\title{
Prader-Willi syndrome
}

INSERM

\section{Source}

INSERM. (1999). Orphanet: an online rare disease and orphan drug data base. Prader-Willi syndrome. ORPHA:739

Prader-Willi syndrome is a rare genetic disorder characterized by hypothalamic-pituitary abnormalities with severe hypotonia during the neonatal period and first two years of life and the onset of hyperphagia with a risk of morbid obesity during infancy and adulthood, learning difficulties and behavioral problems or severe psychiatric problems. 